\title{
Espaces publics et diversité sociale. Introduction au cahier thématique
}

\author{
Elisabeth Bühler, Zurich
}

Les scientifiques ne parviennent guère à s'accorder sur la signification précise de la notion d'espace public (voir Staeheli \& Mitchell 2007). La confusion babylonienne qui entoure ce concept (SELle 2008) peut être expliquée par au moins trois raisons. Premièrement, il n'existe aucune distinction claire entre les termes espace public et sphère publique. Deuxièmement, le terme d'espace public est entouré d'une connotation fortement normative (BELINA 2005), ce qui ne facilite pas la vision analytique des processus qui conduisent à la construction des espaces publics. Troisièmement, il n'existe pas de consensus relatif à la direction prise par le changement de fonction de l'espace public contemporain. Dans les lignes qui suivent, nous revenons brièvement sur chacun de ces trois aspects pour ensuite positionner la contribution de ce cahier thématique par rapport à l'espace public et à la diversité sociale.

\section{Espace public et sphère publique: une clarification de leurs relations}

Dans son ouvrage Raumsoziologie [Sociologie de l'espace] (2001), la sociologue MARTINA Löw a présenté un concept théorique permettant de comprendre le processus de construction des espaces (publics) et de distinguer les termes d'espace public et de sphère publique. Löw considère les espaces comme des constructions sociales qui interviennent dans le cours des actions humaines. Comparées aux autres structures sociales, les structures spatiales ont pour spécificité d'être constituées de composantes physico-matérielles. Löw définit les structures spatiales comme des configurations relationnelles d'objets physico-matériels et de personnes, qui sont reproduites de manière récurrente à travers les actions des acteurs sociaux.

Depuis les Lumières, les sociétés démocratiques n'ont eu de cesse de distinguer sphère publique et sphère privée (SchäFers 2003:15). Selon Löw (2001: 168-172), cette structure sociale fondamentale, garantie par des règles et des ressources, est formée d'un entrelacement de structures sociales isolables et reproduites de manière récurrente. Il s'agit par exemple de structures légales qui permettent de différencier les lois privées (ou civiles) des lois publiques, de structures économiques qui permettent de distinguer le secteur privé (capitaliste) du secteur public, de structures de propriété qui permettent de distinguer la propriété privée de la propriété publique. En admettant avec Löw que les espaces forment des structures sociétales reproduites de manière récurrente, alors les espaces publics et privés ne sont en principe rien d'autre qu'un autre composant isolable dans l'entrelacement structurel entre public et privé. Les espaces publics doivent être compris comme des configurations relationnelles d'objets physico-matériels et de personnes. Concrètement, l'espace public est formé de squares, de rues, de trottoirs, d'espaces verts ou de bâtiments, librement accessibles au public. Dans ces espaces se manifestent différentes autres parties des structures de la sphère publique, parmi lesquelles les structures économiques, juridiques et de propriété.

\section{Les espaces publics comme lieux de participation démocratique}

Dans les sociétés occidentales, un consensus existe pour considérer l'espace comme «public» s'il est accessible à tous, c'est-à-dire si personne n'en est fondamentalement exclu a priori. Cela implique que les règles et les ressources qui interviennent dans la construction des espaces publics doivent se plier aux principes démocratiques. Les conflits d'intérêt entre groupes différents, qui constituent une caractéristique typique des sociétés à forte diversité sociale, doivent être négociés démocratiquement. Les conflits d'usage dans l'espace public doivent alors idéalement être résolus par un consensus. Cependant, comme c'est le cas également dans les politiques publiques, ce consensus n'est généralement pas atteint en ce qui concerne l'espace public, ce qui implique de prendre des décisions sur le principe de la majorité. A titre d'exemple figurent les débats contemporains ayant lieu en Suisse sur le sujet des excès de consommation d'alcool commis par des jeunes ou de la mendicité pratiquée sur la voie publique.

\section{Dans une démocratie, cependant,}

«les opinions majoritaires doivent être justifíes de manière à ce que les minorités puissent accepter le fait qu'elles ont été minorisées. Par conséquent, le processus démocratique doit être organisé de telle sorte que les minorités puissent exprimer leurs intérêts et leurs arguments » (LEUENBERGER 2008: 15, librement traduit).

Dans cette perspective, les jeunes qui consomment de l'alcool en quantité excessive ou les mendiants des rues ne peuvent être purement et simplement expulsés de l'espace public, ce dernier étant compris comme un lieu permettant une participation égalitaire. Il est alors nécessaire d'inclure ces acteurs dans des négociations portant sur les usages de l'espace public, en les considérant comme des partenaires possédant des droits équivalents aux autres usagers. Les principes normatifs permettant d'appréhender ce type de négociations dans les sociétés occidentales ont été énoncés par Fin- 
CHER \& IvESON (2008): redistribution, reconnaissance et rencontre.

Les processus d'exclusion de l'espace public peuvent cependant aussi apparaître de manière plus subtile. Il n'y a pas si longtemps, dans les sociétés occidentales, il était jugé inconvenant pour les femmes de la bourgeoisie de se trouver dans les lieux publics la nuit sans une escorte masculine (Bondi \& Domosh 1998; Ruhne 2003). La raison invoquée était la sécurité des femmes, mais il apparaît clairement que la motivation était le contrôle des femmes par les pères, les maris et les frères. Bien que ces règles aient disparu de nos jours, les espaces publics persistent à être dépeints par les médias comme des espaces potentiellement dangereux pour les femmes, notamment durant la nuit. En conséquence, un grand nombre de femmes se sentent moins en sécurité que les hommes dans l'espace public et continuent à sortir accompagnées, décident de rester à la maison ou évitent certains espaces publics de manière occasionnelle.

Le mythe selon lequel il fut un temps où les espaces publics constituaient des lieux où des acteurs socialement égaux pouvaient se regrouper a été battu en brèche par de nombreux chercheurs (voir notamment Belina 2005; Bondi \& Domosh 1998). Etant donné que les espaces publics peuvent être considérés comme des structures sociales (voir plus haut), ceux-ci sont également façonnés par des relations de pouvoir et de domination. Cette constatation analytique n'exclut toutefois pas que l'orientation normative de l'égalité d'accès soit toujours d'actualité en ce qui concerne la régulation des espaces publics.

\section{La mutation fonctionnelle des espaces publics}

Depuis quelques temps déjà, une attention considérable a été portée par les médias, le monde politique et les chercheurs à la mutation fonctionnelle des espaces publics. Peu de voix s'accordent sur le sens de cette transformation. De manière simplifiée, deux positions opposées peuvent être distinguées:

«la position des sceptiques, qui considèrent le déclin des espaces publics à travers l'exclusion des groupes sociaux les plus faibles, et la position des optimistes, qui observent une renaissance des espaces publics permettant à des groupes plus divers de la société de se mettre en scène» (WIEGandT 2006: 7, librement traduit).

Privatisation et marchandisation sont deux mots-clés qui peuvent être liés à la position des sceptiques, tandis que revitalisation et reconquête peuvent être attribués au groupe des optimistes. Cependant, ces formules ne sont pas des simplifications très utiles de la réalité. Il est bien plus approprié de considérer l'usage et l'accessibilité des espaces publics à travers l'étude d'exemples concrets. C'est l'ambition de ce cahier thématique que de fournir des exemples de ce genre.

\section{Les contributions de ce cahier thématique}

Les cinq articles de ce cahier thématique représentent une (courte) sélection des contributions présentées lors du symposium intitulé «Sustainable Public Spaces» de la Commission Genre et Géographie de l'Union Géographique Internationale (UGI) qui s'est tenue à Zurich du 1er au 3 juin 2007. Les résumés de toutes les contributions du symposium sont téléchargeables sur le site Internet suivant: http:/www.geo. unizh.ch/nfp54/igu07/index.html.

Dans leur article introductif intitulé «Villes des 〈autres〉: espace public et pratiques quotidiennes», Dina Vaiou et Ares Kalandides considèrent quelques-unes des considérations fondamentales des espaces publics dans les sociétés démocratiques à forte diversité sociale mentionnées dans cet éditorial. Les auteurs approfondissent le sujet par l'examen de plusieurs cas d'étude à Berlin et à Athènes et par une discussion critique portant sur les concepts du public et du privé. La contribution de HeIDI KasPar et ELISABETH BÜHLER intitulée «Aménagement, design et usage de l'espace public du Wahlenpark (Zurich, Suisse): ouverture fonctionnelle, visuelle et sémiotique» discute les processus d'inclusion et d'exclusion à partir d'un espace public particulier à la lumière d'une conception relationnelle de l'espace. En se fondant sur une théorie spatiale identique, Frank OsterMANN et SABINE TIMPF montrent que différents groupes produisent différents espaces sur un lieu unique, dans leur contribution intitulée «Usage et appropriation de l'espace dans des parcs publics urbains. Les méthodes SIG en géographie sociale». Les espaces publics forment des observatoires très instructifs pour l'enseignement de la géographie. Joos Droogleever Fortuisn en donne une illustration manifeste dans sa contribution «Observer les différences de genre dans les espaces publics: un outil pour l'enseignement» fondée sur une étude comparative au plan international. La dernière contribution de ce cahier thématique est intitulée «Intersections entre public et privé: jardins communautaires, relations de voisinage et géographie de l'assistance à Buffalo (NY, USA)». Dans cet article, LADona KNIGGE examine de manière critique les concepts de public, de citoyenneté, de genre et de groupe ethnique au travers d'un exemple nordaméricain.

Au total, ces contributions montrent que les catégories dichotomiques espace public/espace privé continuent d'être extrêmement pertinentes dans la vie quotidienne des sociétés occidentales, particulièrement à cause de leur contenu normatif. Cependant, elles ne sont pas très utiles comme catégories analytiques, en raison du fait que, dans les cas concrets, les frontières entre public et privé sont floues et continuellement traversées. Comme le montre la contribution de VAIOU 
et KALANDIDEs, cela est également vrai des dichotomies féminin/masculin, homosexuel/hétérosexuel ou autochtone/étranger.

\section{Bibliographie}

BeLINA, B. (2005): Öffentlich/privat: von strategischen Grenzziehungen in Gesellschaft und Raum. - In: Berichte zur deutschen Landeskunde 79, 2/3: 317-327.

Bondi, L. \& M. Domosh (1998): On the contours of public space: a tale of three women. - In: Antipode 30, 3: 270-289.

FINCHER, R. \& K. Iveson (2008): Planning and diversity in the city. Redistribution, recognition and encounter. - New York: Palgrave Macmillan.

LeUENBERGER, M. (2008): Eigeninteresse und Gemeinwohl in der Politik. Demokratische Legitimierung der Allgemeininteressen. - In: Neue Zürcher Zeitung, 12 juin 2008, Zurich: 15.

Löw, M. (2001): Raumsoziologie. - Frankfurt am Main: Suhrkamp.

Ruhne, R. (2003): Raum Macht Geschlecht. Zur Soziologie eines Wirkungsgefüges am Beispiel von (Un-)Sicherheiten im öffentlichen Raum. - Opladen: Leske+Budrich.
SCHÄFERs, B. (2003): Ansprüche der demokratisch verfassten Gesellschaft an den öffentlichen Raum. - In: Informationen zur Raumentwicklung, cahier thématique «Öffentlicher Raum und Stadtgestalt» 1/2:15-20. SELlE, K. (2008): Spannungsfelder? Öffentliche Räume im Diskurs - und in den Städten. Plätze, Parks und Promenaden. Die Koproduktion der öffentlichen Räume in den Städten. - Aachen: http:/www.pt.rwthaachen.de/content/view/275/107/lang.en/ 23 juin 2008. Staeheli, L.A. \& D. Mrtchell (2007): Locating the public in research and practice. - In: Progress in Human Geography 31, 6: 792-811.

WIEGANDT, C.-C. (2006): Öffentliche Räume - öffentliche Träume. Zur Kontroverse über die Stadt und ihre Gesellschaft. Ein kurzes Vorwort. - In: WiEGandT, C.-C. (éd.): Öffentliche Räume - öffentliche Träume. Zur Kontroverse über die Stadt und ihre Gesellschaft. - Berlin: Lit Verlag: 7-12.

Dr. Elisabeth Bühler, Institut de géographie, Université de Zurich, Winterthurerstrasse 190, CH-8057 Zurich, Suisse.

e-mail: elisabeth.buehler@geo.uzh.ch 